\title{
Orthogonal and diagonal dimension fluxes of hyperspherical function
}

\author{
Dusko Letic ${ }^{1}$, Nenad Cakic ${ }^{2}$, Branko Davidovic ${ }^{3^{*}}$ and Ivana Berkovic ${ }^{4}$
}

\footnotetext{
* Correspondence: iwtbg@beotel. net

${ }^{3}$ Technical High School, Kragujevac, Serbia

Full list of author information is available at the end of the article
}

\begin{abstract}
In this paper, we present the theoretical research results of certain characteristics of the generalized hyperspherical function with two degrees of freedom as independent dimensions. Here, we primarily give the answers to the quantification of dimensional potentials (fluxes) of this function in the domain of natural numbers. In addition, we also give the solutions to continual fluxes of separate contour hyperspherical (HS) functions. The symbolical evaluation and numerical verification of the values of series and integrals are realized using MathCAD Professional and Mathematica.
\end{abstract}

MSC 2010: 51M04; 33E99.

Keywords: special function, hypersphere, dimension flux

\section{Introduction}

The hypersphere function is a hypothetical function related to multi-dimensional space (see [1-3]). The most important aspect of this function is its connection to all functions that describe the properties of spherical entities: points, diameter, circumference, circle, surface, and volume of a sphere. The second property is the generalization of these functions from discrete to continuous. It belongs to the group of special functions, so its testing is being performed on the basis of known functions such as gamma $(\Gamma)$, psi $(\psi)$, and the like, so that its generalized, explicit form is the following [4].

Definition 1.1. The hyperspherical function [5] with two degrees of freedom $k$ and $n$ is defined as

$$
H S(k, n, r)=\frac{2 \sqrt{\pi^{k}} r^{k+n-3} \Gamma(k)}{\Gamma(k+n-2) \Gamma\left(\frac{k}{2}\right)} \quad(k, n \in Z, r \in N),
$$

where $\Gamma(\mathrm{z})$ is the gamma function.

Using the fundamental properties of the gamma function, we advance from the domain of the natural values analytically to the set of real values for which we form the conditions for both its graphical interpretation and a more concise mathematical analysis. It is developed on the basis of two degrees of freedom $k$ and $n$ as vector dimensions, in addition to radius $r$, as an implied degree of freedom for every

(c) 2012 Letic et al; licensee Springer. This is an Open Access article distributed under the terms of the Creative Commons Attribution License (http://creativecommons.org/licenses/by/2.0), which permits unrestricted use, distribution, and reproduction in any medium, provided the original work is properly cited. 
hypersphere. The dominant theorem is the one that relates to the recurrent property of this function [6].

It implies that the vectors on the left $(n=2,1,0,-1,-2, \ldots)$ of the matrix $M[H S]_{k x n}$ (1.3) are obtained on the base of the reverent vector $(n=3)$ deduction, and the vectors on the right $(n=4,5,6,7,8, \ldots)$ on the base of integrals by radius $r$ [7].

$$
\frac{\partial}{\partial r} H S(k, n, r)=H S(k, n-1, r) \text { and } H S(k, n+1, r)=\int_{0}^{r} H S(k, n, r) d r .
$$

For the development of the hyperspherical functions theory see Bishop [8], Conway [1], Dodd and Coll [2], Hinton [9], Hocking and Young [10], Manning [3], Maunder [11], Neville [12], Rohrmann and Santos [13], Satoshi et al. [14], Sloane [15], Rucker [16], Sommerville [17], Weels [18], Joshi and Sadan [19], Kabatiansky [20], Letić and all [21], Loskot and Norman [22], Sasaki [23], Tu and Fischbach [24], Woonchul and Zhou [25], and for its testing, see Ramanujan and Hardy [26]. Today the research of hyperspherical functions is represented both in Euclid's and Riemann's geometries and topologies (Riemann's and Poincare's spheres, multidimensional potentials, theory of fluids, atomic physics, hyperspherical black holes, so on) (Figures 1, 2).

$$
M[H S]_{k x n}=
$$

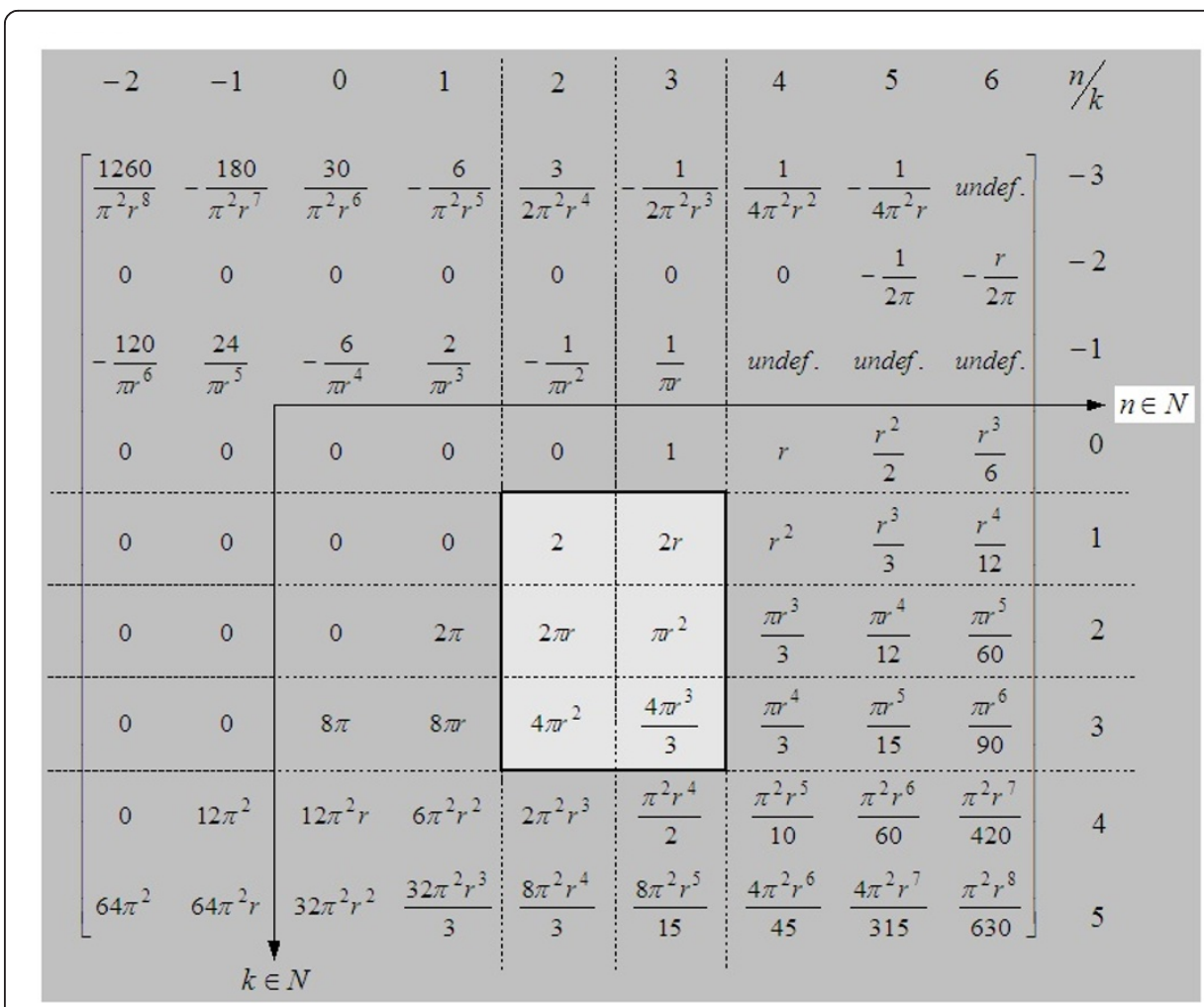

Figure 1 The submatrix $H S(k, n, r)$ of the function for $k L-3,-2, \ldots .5$ and $n L-2,-1, \ldots 6$ with six highlighted characteristics functions (undef. are undefined values, most commonly singular $\pm \infty$ of this function value). 


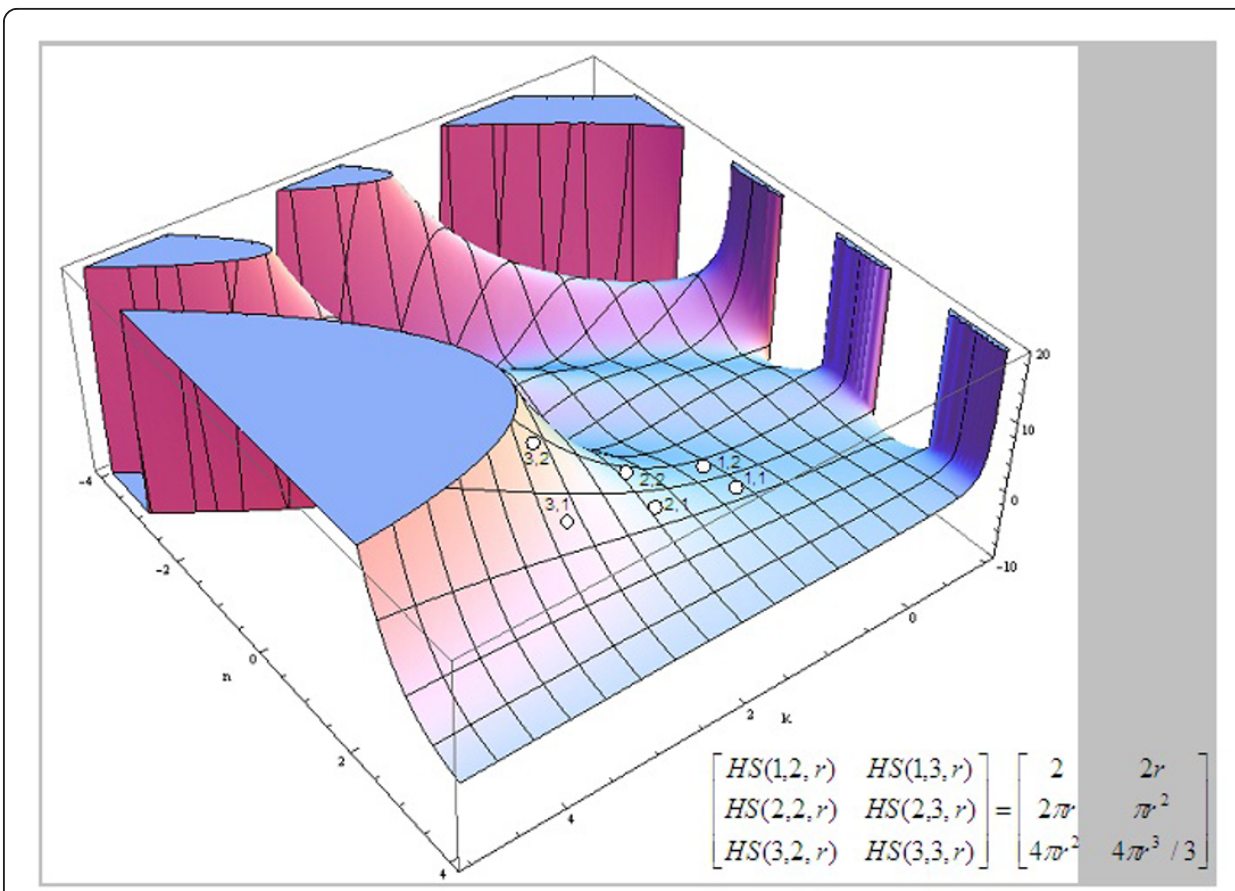

Figure 2 The 3D hyperspherical function for the unitary radius $H S(-1 \leq k \leq 5,-4 \leq n \leq 4,1)$ and the coordinates of real spherical entities.

\section{Dimensional potentials-the fluxes of $H S$ function}

\subsection{Vertical dimensional flux of hypersphere function}

Definition 2.1. The discrete dimensional potential or the hypersphere function flux is the sum of all separate functions in the (sub)matrix of this function that we expand for integer or real degrees of freedom (1.3).

Formally, this flux can be quantified by twofold series that covers this area of the $H S$ function. The first phase is to define the value of infinite series of functions classified in columns (vectors) of the submatrix $M[H S]_{k x n}(k, n \in N)$. This is also the definition of vertical dimensional fluxes of $H S$ function. The first value to be calculated relates to the fourth columns $(n=3)$ of this submatrix (1.3). From this, we obtain

$$
\sum_{k=0}^{\infty} H S(k, 3, r)=1+2 r+\pi r^{2}+\frac{4}{3} \pi r^{3}+\frac{1}{2} \pi^{2} r^{4}+\frac{8}{15} \pi^{2} r^{5}+\cdots+\varepsilon\left(\frac{2 \sqrt{\pi^{k}} r^{k}}{k \Gamma(k / 2)}\right) .
$$

Freden [27] has precisely defined this series with the value

$$
\sum_{k=0}^{\infty} H S(k, 3, r)=\sum_{k=0}^{\infty} \frac{2 \sqrt{\pi^{k}} r^{k}}{k \Gamma(k / 2)}=e^{\pi r^{2}}[1+\operatorname{erf}(r \sqrt{\pi})]
$$

where $\operatorname{erf}(z)$ is an error function. When $k$ is even $(0,2,4, \ldots)$, respectively, odd $(1,3,5, \ldots)$, that series can be classified as dichotomous, so we obtain two complementary series

$$
\sum_{k=0}^{\infty} H S(k, 3, r)=\sum_{k=0,2,4, \ldots}^{\infty} H S(k, 3, r)+\sum_{k=1,3,5, \ldots}^{\infty} H S(k, 3, r)=e^{\pi r^{2}}+e^{\pi r^{2}} \operatorname{erf}(r \sqrt{\pi})
$$


Consequently, Freden's result [27] can be presented in the form of series with even $(k=2 b)$ and odd members $(k=2 b+1$, where $b \in N)$. In this sense we get

$$
e^{\pi r^{2}}=\sum_{b=0}^{\infty} \frac{\pi^{b} r^{2 b}}{b !} \quad \text { and } \quad e^{\pi r^{2}} \operatorname{erf}(r \sqrt{\pi})=\sum_{b=0}^{\infty} \frac{\pi^{b}(2 r)^{2 b+1} b !}{(2 b+1) !} .
$$

On the base of Freden's solution (2.1), as a starting point and applying the recurrent relations (1.2), we get values for lower degrees of freedom $(n<3)$. We connect the sphere hypervolume $(n=3)$ with its hypersurface $(n=2)$. In this sense a new vector flux follows

$$
\frac{\partial}{\partial r} \sum_{k=0}^{\infty} H S(k, 3, r)=\sum_{k=0}^{\infty} H S(k, 2, r) \text {, so then is } \sum_{k=0}^{\infty} H S(k, 2, r)=2\left[1+\pi r e^{\pi r^{2}} \operatorname{erfc}(-r \sqrt{\pi})\right] .
$$

For hypersphere $(n=1)$, a series is obtained in view of the previous, therefore

$$
\frac{\partial}{\partial r} \sum_{k=0}^{\infty} H S(k, 2, r)=\sum_{k=0}^{\infty} H S(k, 1, r)=2 \pi\left[2 r+e^{\pi r^{2}}\left(1+2 \pi r^{2}\right) \operatorname{erfc}(-r \sqrt{\pi})\right]
$$

For $n=0$, the series value is found on the basic of deducing, so it follows that

$$
\sum_{k=0}^{\infty} H S(k, 0, r)=8 \pi^{2}\left[r e^{\pi r^{2}}\left(\frac{3}{2}+\pi r^{2}\right) e r f c(-r \sqrt{\pi})+r^{2}+\pi^{-1}\right] .
$$

For degrees of freedom higher than $n=3$, series are found by inverse operations, i.e., by recurrent relation in view of integrating along radius $r$. Consequently,

$$
\sum_{k=0}^{\infty} H S(k, n+1, r)=\int_{0}^{r}\left(\sum_{k=0}^{\infty} H S(k, n, r)\right) d r
$$

so for the fourth dimension the following integral form is applied

$$
\sum_{k=0}^{\infty} H S(k, 4, r)=\int_{0}^{r}\left(\sum_{k=0}^{\infty} H S(k, 3, r)\right) d r=\int_{0}^{r} e^{\pi r^{2}}[1+\operatorname{erf}(r \sqrt{\pi})] d r .
$$

This property refers also to the complementary dichotomous hyperspherical series. In that case, we have for even members

$$
\sum_{k=0,2,4, \ldots}^{\infty} H S(k, 4, r)=\int_{0}^{r}\left(\sum_{k=0,2,4, \cdots}^{\infty} H S(k, 3, r)\right) d r
$$

So for the fourth dimension an integral form with imaginary error function is obained using $\operatorname{erf}(z)=-\operatorname{ierf}(i z)$.

$$
\sum_{k=0,2,4, \ldots}^{\infty} H S(k, 4, r)=\int_{0}^{r} e^{\pi r^{2}} d r=-\frac{i}{2} \operatorname{erf}(r \sqrt{-\pi})=\frac{\operatorname{erfi}(r \sqrt{\pi})}{2} .
$$

After the partial integration using $\int u d v=u v-\int v d u$ we obtain the integral for "odd" series 


$$
\sum_{k=1,3,5, \ldots}^{\infty} H S(k, 4, r)=\int_{0}^{r} \operatorname{erf}(r \sqrt{\pi}) e^{\pi r^{2}} d r=\frac{\operatorname{erff}(r \sqrt{\pi}) \operatorname{erf}(r \sqrt{\pi})}{2}-\int_{0}^{r} e^{-\pi r^{2}} \operatorname{erff}(r \sqrt{\pi}) d r,
$$

where $u=\operatorname{erf}(r \sqrt{\pi})$ and $d v=e^{\pi r^{2}} d r$. The addend with the integral on the right side is analytically solvable and it amounts to, e.g., the series with " $b$ " members (or as a series with the incomplete gamma function). In that sense, the value of the series with even numbers is

$$
\sum_{k=0,2,4, \ldots}^{\infty} H S(k, 4, r)=\sum_{k=0,2,4, \ldots}^{\infty} \frac{\sqrt{\pi^{k}} r^{k+1}}{(k+1)(k / 2) !}=\sum_{b=0}^{\infty} \frac{\pi^{b} r^{1+2 b}}{(2 b+1) b !},
$$

while the series for odd numbers is defined as

$$
\sum_{k=1,3, \ldots}^{\infty} H S(k, 4, r)=\sum_{k=1,3, \ldots}^{\infty} \frac{2^{k} \sqrt{\pi^{k-1}} r^{k+1}}{(1+k) !}\left(\frac{k-1}{2}\right) ! \equiv \sum_{b=0}^{\infty} \frac{2^{1+2 b} \pi^{b} r^{2(1+b)} b !}{(2+2 b) !} .
$$

So, the analytical values of these dichotomous series are

$$
\int_{0}^{r} e^{\pi r^{2}}[1+e r f(r \sqrt{\pi})] d r=\int_{0}^{r} e^{\pi r^{2}} d r+\int_{0}^{r} e^{\pi r^{2}} \operatorname{erf}(r \sqrt{\pi}) d r=\frac{e r f(r \sqrt{\pi})}{2}+\sum_{b=0}^{\infty} \frac{2^{1+2 b} \pi^{b} b ! r^{2(1+b)}}{(2+2 b) !} .
$$

\subsection{Integral solvability on the base of the incomplete gamma function}

The flux for $n=3$ is the easiest one to solve, and it represents the base for calculating fluxes of higher degrees of freedom $(n>0)$, through integration of previously obtained results. In that sense, this procedure is possible by using the series where the incomplete gamma function. The second integral (2.2) is reduced to known terms, and one among them is [28]

$$
\int e^{b z^{2}} \operatorname{erf}(a z) d z=\frac{1}{b \sqrt{\pi}} \sum_{k=0}^{\infty} \frac{a^{2 k+1} \Gamma\left(k+1,-b z^{2}\right)}{b^{k}(2 k+1) k !}+C .
$$

Integral in its definite form is expressed as

$$
\int_{0}^{r} e^{\pi r^{2}}[1+e r f(r \sqrt{\pi})] d r=\frac{\operatorname{erfi}(r \sqrt{\pi})}{2}+\frac{1}{\pi} \sum_{k=0}^{\infty} \frac{\Gamma\left(k+1,-\pi r^{2}\right)-\Gamma(k+1,0)}{(1+2 k) k !} .
$$

While the incomplete gamma function is in general case equal to $\Gamma(a, z)=\int_{z}^{\infty} t^{a-1} e^{-t} d t[29]$. It is obvious that the two obtained series with odd members are equivalent to the following

$$
\frac{1}{\pi} \sum_{k=0}^{\infty} \frac{\Gamma\left(k+1,-\pi r^{2}\right)-\Gamma(k+1)}{(1+2 k) k !} \equiv \sum_{b=0}^{\infty} \frac{2^{1+2 b} \pi^{b} r^{2(1+b)} b !}{(2+2 b) !} .
$$


Defining the series for $n=5$ is realized by integrating the expressions

$$
\sum_{k=0}^{\infty} H S(k, 5, r)=\int_{0}^{r} \frac{\operatorname{erfi}(r \sqrt{\pi})}{2} d r+\int_{0}^{r}\left(\sum_{k=1,3, \ldots}^{\infty} \frac{2^{k} \sqrt{\pi^{k-1}} r^{k+1}}{(1+k) !}\left(\frac{k-1}{2}\right) !\right) d r
$$

The first integral on the right side of (2.3) is solved on the bases of the known equality $\int \operatorname{erfi}(a z)=z \cdot \operatorname{erfi}(a z)-\frac{e^{(a z)^{2}}}{a \sqrt{\pi}}$, where $a z=r \sqrt{\pi}$. Thus, the integral is obtained as

$$
\int_{0}^{r} \frac{\operatorname{erfi}(r \sqrt{\pi})}{2} d r=\left.\frac{1}{2}\left(r \cdot \operatorname{erfi}(r \sqrt{\pi})-\frac{e^{\pi r^{2}}}{\pi}\right)\right|_{0} ^{r}=\frac{1-e^{\pi r^{2}}+\pi \operatorname{rerfi}(r \sqrt{\pi})}{2 \pi} .
$$

However, a simpler way is to use both even and odd dichotomous series. Now, we obtain

$$
\sum_{k=0,2, \ldots}^{\infty} H S(k, 5, r)=\int_{0}^{r}\left(\sum_{b=0}^{\infty} \frac{\pi^{b} r^{2 b+1}}{(2 b+1) b !}\right) d r=\frac{1}{2} \sum_{b=0}^{\infty} \frac{\pi^{b} r^{2(b+1)}}{(2 b+1)(b+1) !}
$$

respectively

$$
\sum_{k=1,3, \ldots}^{\infty} H S(k, 5, r)=\int_{0}^{r}\left(\sum_{b=0}^{\infty} \frac{2^{1+2 b} \pi^{b} b ! r^{2(b+1)}}{(2+2 b) !}\right) d r=\sum_{b=0}^{\infty} \frac{2^{1+2 b} \pi^{b} b ! r^{2 b+3}}{(3+2 b) !} .
$$

The sum of results on the base of the integral value and one series is

$$
\sum_{k=0}^{\infty} H S(k, 5, r)=\frac{1-e^{\pi r^{2}}+\pi r e r f i(r \sqrt{\pi})}{2 \pi}+\sum_{b=0}^{\infty} \frac{2^{1+2 b} \pi^{b} b ! r^{2 b+3}}{(3+2 b) !}
$$

or with two complementary series

$$
\sum_{k=0}^{\infty} H S(k, 5, r)=\sum_{k=1}^{\infty} \frac{\sqrt{\pi^{k}} r^{k+2}}{(k+1)(k+2) \Gamma\left(\frac{k}{2}+1\right)}=\frac{1}{2} \sum_{b=0}^{\infty} \frac{\pi^{b} r^{2(b+1)}}{(2 b+1)(b+1) !}+\sum_{b=0}^{\infty} \frac{2^{1+2 b} \pi^{b} b ! r^{2 b+3}}{(3+2 b) !} .
$$

The dichotomous series for $n=6$ is

$$
\sum_{k=0}^{\infty} H S(k, 6, r)=\sum_{k=0}^{\infty} \frac{\sqrt{\pi^{k} r^{k+3}} \Gamma(k+1)}{\Gamma(k+3) \Gamma\left(\frac{k}{2}+1\right)}=\sum_{b=0}^{\infty} \frac{2^{1+2 b} \pi^{b} b ! r^{2(b+2)}}{(4+2 b) !}+\frac{1}{2} \sum_{b=0}^{\infty} \frac{\pi^{b} r^{2 b+3}}{(2 b+1)(2 b+3)(b+1) !} .
$$

Note: Integration, similar as in the previous cases, is applied with certain conditions, so we have, e.g., $\int_{0}^{r} r^{2 b+3} d r=0$, if $-1<2 \operatorname{Re}(b)+3<-1^{\wedge} \mathrm{b} \neq-1^{\wedge} r=\infty$. If the conditions are not met, this integral is indefinite. Some values of these discrete and continuous fluxes (for $r=1$ ) are given in Table 1 .

Suppose that the values of the vector fluxes decline with the increase of the degree of freedom $n$. The dimensional fluxes can be studied as well for the complex part. So, for example, with the recurrence we get the series values for the negative degree of 
Table 1 Values of discrete and continuous fluxes.

\begin{tabular}{lll}
\hline Degree of freedom $(n)$ & $\sum_{k=0}^{\infty} H S(k, n, 1) \approx$ & $\int_{0}^{\infty} H S(k, n, 1) d k \approx$ \\
\hline 0 & 16962.1740457 & 16962.3520362 \\
1 & 2117.56926532 & 2117.48007283 \\
2 & 291.022289825 & 291.104223905 \\
3 & 45.9993260894 & 45.5712471365 \\
4 & 8.71952109668 & 8.20993584833 \\
5 & 1.87596579993 & 1.60128605246 \\
6 & 0.40326040109 & 0.30739217922 \\
7 & 0.07910676340 & 0.05435115208 \\
8 & 0.01367865325 & 0.00860415949 \\
9 & 0.00207449183 & 0.00121196056 \\
10 & 0.00027764247 & 0.00015240808 \\
11 & 0.00003309744 & 0.00001722662 \\
12 & 0.00000354778 & 0.00000176333 \\
$\vdots$ & $\vdots$ & $\vdots$ \\
$\infty$ & $\lim _{n \rightarrow \infty}^{\infty} \sum_{k=0}^{\infty} H S(k, n, r)=0$ & $\lim \int_{n \rightarrow \infty}^{\infty} H S(k, n, r) d k=0$ \\
$\Sigma$ & & \\
$n$ & 19427.858848843922 & - \\
\hline & & \\
\hline
\end{tabular}

freedom $n=-2$, as [30].

$$
\sum_{k=0}^{\infty} H S(k,-2, r)=8 \pi^{3}\left\{r e^{\pi r^{2}} \operatorname{erfc}(-r \sqrt{\pi})\left[15+4 \pi r^{2}\left(5+\pi r^{2}\right)\right]+2 r^{2}\left(9+2 \pi r^{2}\right)+8 \pi^{-1}\right\} .
$$

\subsection{Fluxes on the base of hypersphere matrix series}

The discrete dimensional fluxes can be calculated as well "horizontally", i.e., by adding function values along the $M[H S]_{k x n}$ submatrix series. For example, by expanding the series for $k=3$, the flux would contain the following members

$$
\sum_{n=0}^{\infty} H S(3, n, r)=8 \pi+8 \pi r+4 \pi r^{2}+\frac{4}{3} \pi r^{3}+\frac{1}{3} \pi r^{4}+\frac{1}{15} \pi r^{5}+\ldots+\varepsilon\left(\frac{8 \pi r^{n}}{\Gamma(n+1)}\right) .
$$

Some values of discrete and continual fluxes, (for $r=1$ ), are given in Table 2 .

\subsection{Some continuous fluxes}

The distribution trend of vector fluxes is increasing, followed by asymptotic decrease with linear growth of degree of freedom $n$. From the standpoint of functional analysis, the most interesting series of the matrix $M[H S]_{k, n}$ is the one that relates to the degrees of freedom $k=2$ and $k=3$. The first series includes the known functions for the circumference $(2 \pi r)$ and the surface of circle $\left(\pi r^{2}\right)$. The members of the second series are the surface functions $\left(4 \pi r^{2}\right)$ and sphere volume $\left(\frac{4}{3} \pi r^{3}\right)$. The same series are interesting as well for continuous fluxes. The continuous natural flux for the 
Table 2 Values of discrete and continual fluxes

\begin{tabular}{|c|c|c|c|}
\hline $\begin{array}{l}\text { Degree of freedom } \\
(k)\end{array}$ & $\sum_{n=0}^{\infty} H S(k, n, r)=$ & $\sum_{n=0}^{\infty} H S(k, n, 1) \approx$ & $\int_{0} H S(k, n, 1) d n \approx$ \\
\hline 0 & $e^{r}$ & 2.71828182846 & 2.89982256317 \\
\hline 1 & $2 e^{r}$ & 5.43656365692 & 5.24809906025 \\
\hline 2 & $2 \pi e^{r}$ & 17.0794684453 & 17.6417407306 \\
\hline 3 & $8 \pi e^{r}$ & 68.3178737814 & 56.964225268 \\
\hline 4 & $12 \pi^{2}\left(e^{r}-1\right)$ & 203.505142758 & 139.918441638 \\
\hline 5 & $64 \pi^{2}\left(e^{r}-r-1\right)$ & 453.706079704 & 271.32230045 \\
\hline 6 & $60 \pi^{3}\left[2 e^{r}-(r+1)^{2}-1\right]$ & 812.172812098 & 437.960809928 \\
\hline 7 & $\sum_{n=0}^{\infty} \frac{768 \pi^{3} r^{n+4}}{\Gamma(n+5)}$ & 1229.10258235 & 611.722905550 \\
\hline 8 & $\sum_{\substack{n=0 \\
\infty}}^{\infty} \frac{1680 \pi^{4} r^{n+5}}{\Gamma(n+6)}$ & 1628.04409715 & 759.633692941 \\
\hline 9 & 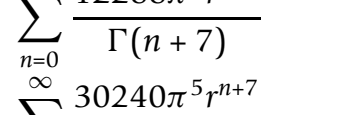 & 1933.28876014 & 855.051695653 \\
\hline 10 & $\begin{array}{l}\sum_{\substack{n=0 \\
\infty}} \frac{\Gamma(n+8)}{245760 \pi^{5} r^{n+8}}\end{array}$ & 2093.93742907 & 884.975895298 \\
\hline 11 & $\begin{array}{c}\sum_{\substack{n=0 \\
\infty}} \frac{\Gamma(n+9)}{665280 \pi^{6} r^{n+9}}\end{array}$ & 2095.29352414 & 851.441651487 \\
\hline 12 & $\begin{array}{l}\sum_{n=0} \frac{}{\Gamma(n+10)} \\
\sum^{\infty} 5898240 \pi^{6} r^{n+10}\end{array}$ & 1956.27052708 & 768.011397877 \\
\hline 13 & $\sum_{n=0} \frac{}{\Gamma(n+11)}$ & 1717.51550066 & 653.938458847 \\
\hline$\vdots$ & & $\vdots$ & $\vdots$ \\
\hline 50 & $\sum_{n=0} H S(50, n, r)$ & 0.00000002078 & 0.000000000526 \\
\hline$\vdots$ & $\vdots$ & $\vdots$ & $\vdots$ \\
\hline$\infty$ & $\lim _{k \rightarrow \infty} \sum_{n=0}^{\infty} H S(k, n, r)=0$ & 0 & $\lim _{k \rightarrow \infty} \int_{0}^{\infty} H S(k, n, r) d n=0$ \\
\hline $\begin{array}{l}\sum_{k} \\
\underline{L}\end{array}$ & & 19427.858848843922 & - \\
\hline
\end{tabular}

hypersphere surface is analyzed on the base of integrals, instead of series. This integral is specific, because its subintegral function is the reciprocal gamma function. Its value, as it is known, is equal to the value of Fransen-Robinson constant [31]

$$
F=\int_{0}^{\infty} \frac{1}{\Gamma(x)} d x=e+\int_{0}^{\infty} \frac{e^{-n}}{\pi^{2}+\ln ^{2} n} d n \approx 2.8077702420285
$$


(a)

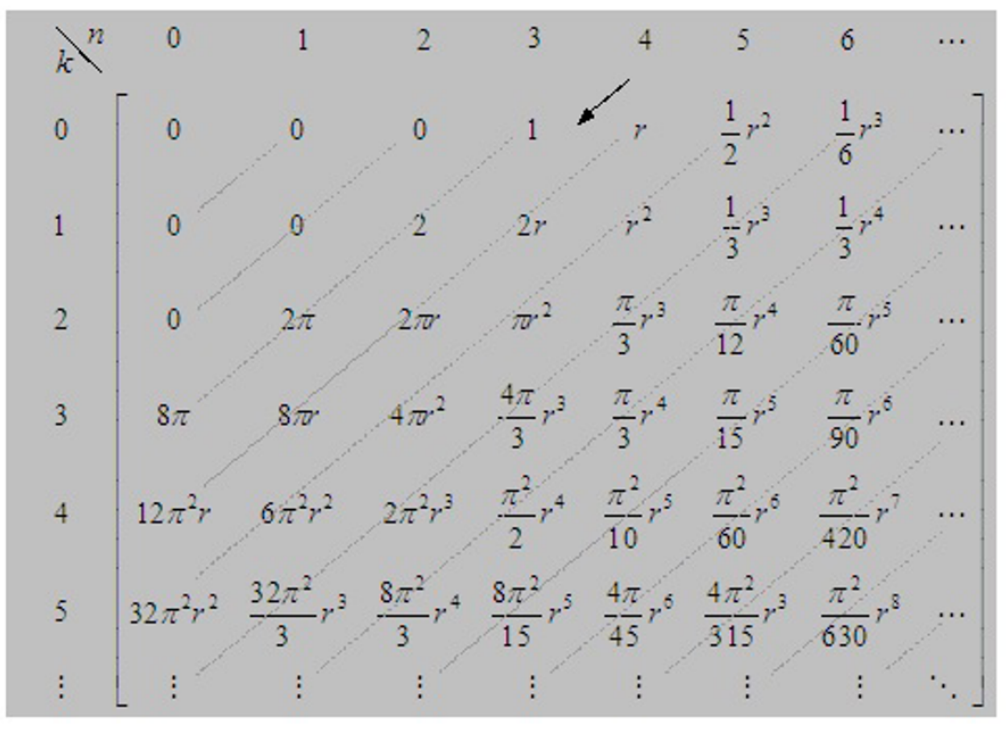

(b)

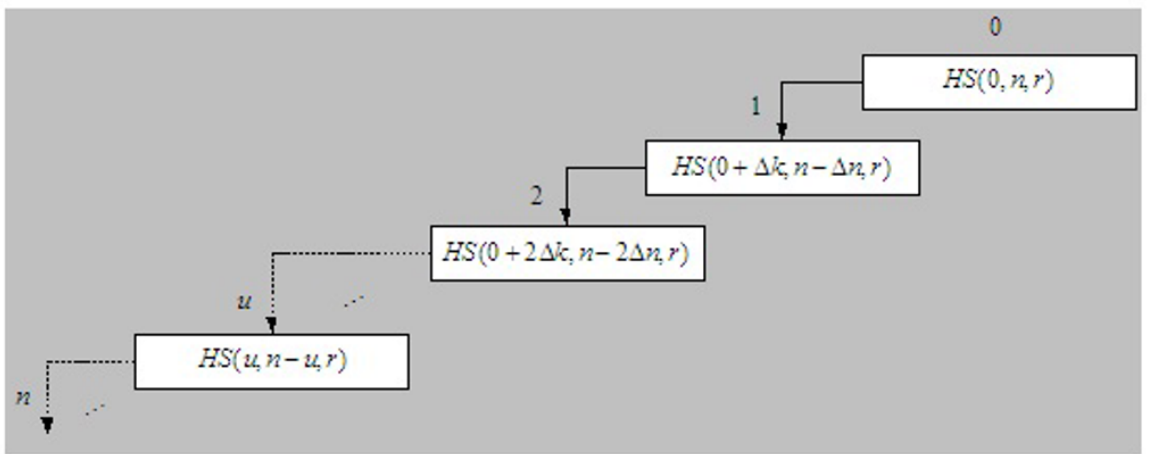

Figure 3 (a) The representative submatrix. Figure 3. (b) The addition principle of the matrix on diagonal.

The integral value of the flux in question is now $\int_{0}^{\infty} H S(2, n, 1) d n=\sum_{n=0}^{\infty} H S(2, n, 1)+\int_{0}^{\infty} \frac{2 \pi e^{-n}}{\pi^{2}+\ln ^{2} n} d n$ or, namely, for unit radius

$$
\int_{0}^{\infty} \frac{2 \pi}{\Gamma(n)} d n=2 \pi\left(e+\int_{0}^{\infty} \frac{e^{-n}}{\pi^{2}+\ln ^{2} n} d n\right)=2 \pi F \approx 17.641741
$$

Regarding the continuous dimension $n$, a more general dimensional volume hypersphere flux follows on the base of Ramanujan-Hardy's integral [26].

$$
\int_{0}^{\infty} \frac{y^{x}}{\Gamma(x+1)} d x=e^{x}-\int_{0}^{\infty} \frac{e^{-x z}}{x\left(\pi^{2}+\ln ^{2} x\right)} d x
$$


Ramanujan defined this integral and Hardy "deepened" it analytically. In that sense, the previous expression can be applied on flux calculation, as

$$
\int_{0}^{\infty} \frac{8 \pi r^{n}}{\Gamma(n+1)} d n=8 \pi\left(e^{r}-\int_{0}^{\infty} \frac{e^{-n r}}{n\left(\pi^{2}+\ln ^{2} n\right.} d n\right) .
$$

The integral can be defined as the difference of series and integral with the value (for $r=1)$

$$
\int_{0}^{\infty} \frac{8 \pi r^{n}}{\Gamma(n+1)} d n=\sum_{n=0}^{\infty} \frac{8 \pi r^{n}}{\Gamma(n+1)}-\left.\int_{0}^{\infty} \frac{8 \pi e^{-n r}}{n\left(\pi^{2}+\ln ^{2} n\right)} d n\right|_{r=1} \approx 56.96423 .
$$

\subsection{Vector flux series}

Total dimensional flux of the degree of freedom in the domain of natural numbers is obtained as the result of twofold amount by which integer values of hyperspherical function $H S(k, n, r),(k, n, r \geq 0)$ are respected. This twofold series has to be convergent, and this property is in the function of hypersphere radius. As usual, calculating of total discrete flux is being performed with its unit value and the convergence is in that case provided, taking into consideration that the unit series on that condition are convergent. The flux can be considered also for each column $M[H S]_{k, n}$ of the matrix, separately. So, we have for the $n$th column (denoted by $<n>$ ), the flux in the following form

$$
\Phi_{H S}^{<n>}(k, n, r)=\sum_{k=0}^{\infty} H S(k, n, r)
$$

\subsection{Orthogonal dimensional flux}

These fluxes are all columns or $M[H S]_{k, n}$. As the number of columns, respectively, series, is infinite, we introduce the following definition for the total flux.

Definition 2.1. The dimensional flux of the functional matrix with two degrees of freedom $k$ and $n$ is defined as a double series

$$
\Phi_{H S}(k, n, r)=\sum_{n=0}^{\infty} \Phi_{H S}^{<n>}(k, n, r)=\sum_{n=0}^{\infty} \sum_{k=0}^{\infty} H S(k, n, r) .
$$

As the defined number of members is calculated, the flux has the form

$$
\begin{aligned}
& \Phi_{H S}(k, n, r)=\sum_{n=0}^{\infty} \sum_{k=0}^{\infty} H S(k, n, r)=8 \pi^{2}\left[r e^{\pi r^{2}}\left(3 / 2+\pi r^{2}\right) \operatorname{erfc}(-r \sqrt{\pi})+r^{2}+\pi^{-1}\right] \\
& +2 \pi\left[2 r+e^{\pi r^{2}}\left(1+2 \pi r^{2}\right) \operatorname{erfc}(-r \sqrt{\pi})\right]+2\left[1+\pi r e^{\pi r^{2}} \operatorname{erfc}(-r \sqrt{\pi})\right]+e^{\pi r^{2}} \operatorname{erfc}(-r \sqrt{\pi}) \\
& +\frac{\operatorname{erf}((r \sqrt{\pi})}{2}+\sum_{b=0}^{\infty} \frac{2^{1+2 b} \pi^{b} b ! r^{2(1+b)}}{(2+2 b) !}+\frac{1-e^{\pi r^{2}}+\pi r \operatorname{erfi}(r \sqrt{\pi})}{2 \pi}+\sum_{b=0}^{\infty} \frac{2^{1+2 b} \pi^{b} b ! r^{2 b+3}}{(3+2 b) !}+\cdots
\end{aligned}
$$

The flux along the matrix series in the domain of natural numbers is defined as a twofold series, but with the summing order changed. This dimensional flux is defined as (2.3.4) 


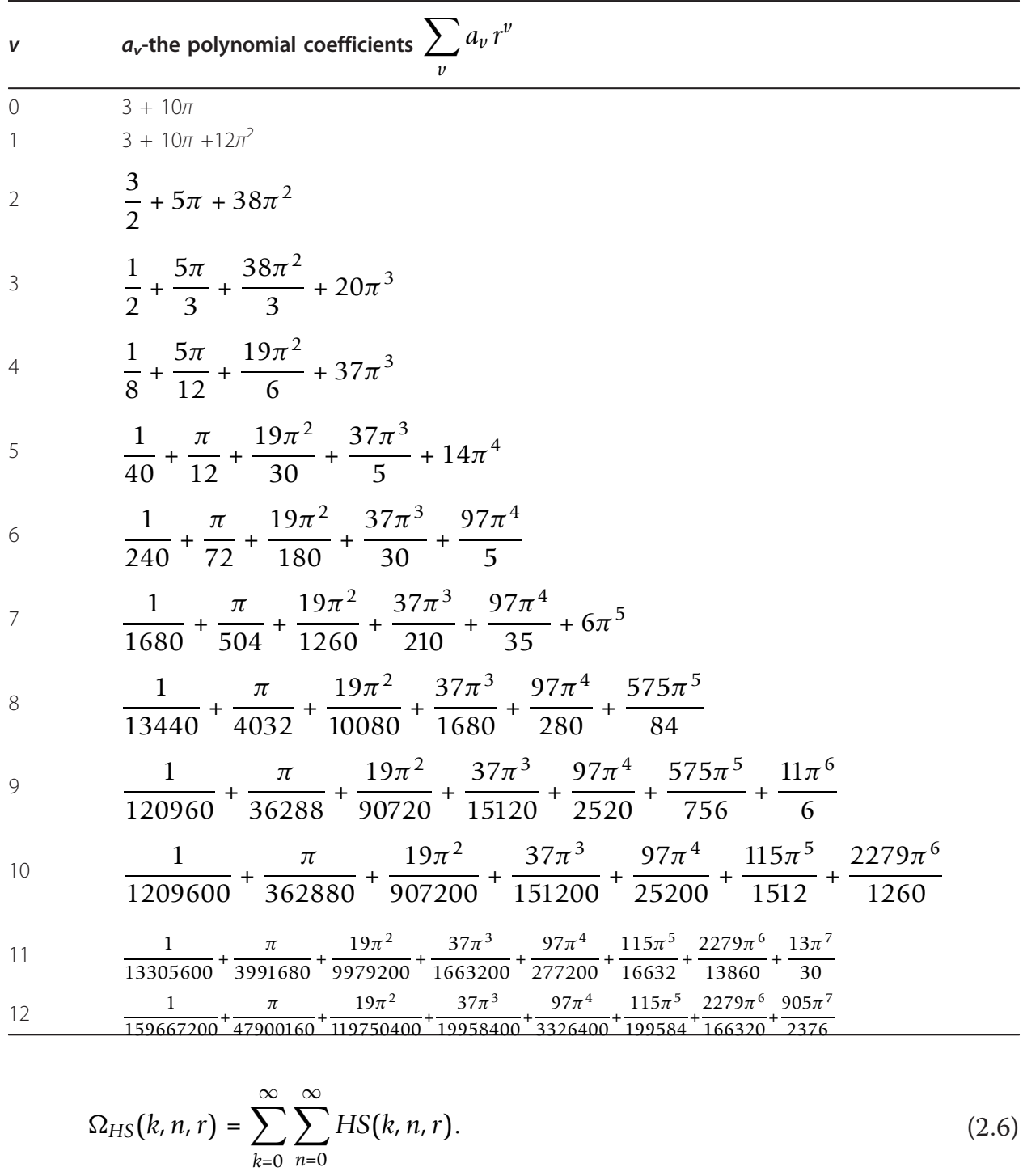

On the basis of the previously fixed members, the matrix flux has the form

$$
\begin{aligned}
& \Omega_{H S}(k, n, r)=\sum_{k=0}^{\infty} \sum_{n=0}^{\infty} H S(k, n, r)=e^{r}+2 e^{r}+2 \pi e^{r}+8 \pi e^{r}+12 \pi^{2}\left(e^{r}-1\right)+64 \pi^{2}\left(e^{r}-r-1\right) \\
& +60 \pi^{3}\left[2 e^{r}-(r+1)^{2}-1\right]+128 \pi^{3}\left[6\left(e^{r}-r-1\right)-r^{2}(r+3)\right] \\
& +70 \pi^{4}\left[24\left(e^{r}-r-1\right)-r^{2}\left(r^{2}+4 r+12\right)\right]+\sum_{n=0}^{\infty} \frac{12288 \pi^{4} r^{n+6}}{\Gamma(n+7)}+\cdots
\end{aligned}
$$

The equivalency of orthogonal dimensional fluxes implies equality of twofold series

$$
\Phi_{H S}(k, n, r)=\Omega_{H S}(k, n, r) .
$$

So, e.g., for $r=1$ dimensional fluxes have unambiguous numerical value

$$
\Phi_{H S}(k, n, 1)=\Omega_{H S}(k, n, 1) \approx 19427.85884884322 .
$$




\subsection{The application of the recurring operators at defining diagonal dimension fluxes}

In the previous analysis, the defining of the dimensional fluxes of the $H S$ matrix was performed on the basis of addition of the $H S$ function values on the columns, in regard to the series of the HS matrix. The more detailed analysis would be very large scale, including the exponential function, error functions $\operatorname{erf}(z), \operatorname{erf}(z)$, the incomplete gamma function $\Gamma(a, z)$, etc. When we use the idea of the transition operators from the reference function into the defining $H S$ function in the functional hyperspherical matrix, we can also establish the values of the dimensional fluxes on the diagonals (Figure 3), whose sum would present the overall flux for the matrix where the degree of freedom is in the domain of natural numbers, i.e., $k, n \in N$. Such matrix contains an infinite number of elements. For the reference functions, we take $H S$ functions on the positions of the first series of the matrix, and they are the so-called zero $H S$ functions: $H S$ $(0,0, r), H S(0,1, r), \ldots, H S(0, n, r), \ldots$ The defining functions are placed according to the "gradual" law of growth $(+\Delta k)$ and decline $(-\Delta n)$.

Definition 2.2. The flux operator of the series $\vartheta(\Delta k, \Delta n, 0)$ is defined by the quotient [4]

$$
\vartheta(\Delta k, \Delta n, 0)=\frac{H S(k+\Delta k, n+\Delta n, r)}{H S(k, n, r)}=\frac{\sqrt{\pi^{\Delta k}} r^{\Delta k+\Delta n} \Gamma(k+n-2) \Gamma(k+\Delta k)}{\Gamma(k) \Gamma(k+n+\Delta k+\Delta n-2) \Gamma\left(\frac{k+\Delta k}{2}\right)} \Gamma\left(\frac{k}{2}\right) .
$$

Also as the absolute values of the increments are equal and unique, that is $|\Delta k|=$ $|-\Delta n|=1$, a new joint argument $u(\Delta k=\Delta n=u)$ is assigned to them. In addition to the starting value of the $k$ th degree of freedom is $k=0$, the operator theta becomes

$$
\theta(u,-u, 0)=2 \sqrt{\pi^{u}} \frac{\Gamma(u)}{\Gamma(u / 2)} .
$$

The assigning function is now being calculated as

$$
H S(u, n-u, r)=\theta(u,-u, 0) \cdot H(0, n, r)=\frac{2 \sqrt{\pi^{u}} r^{n-3} \Gamma(u)}{\Gamma(n-2) \Gamma(u / 2)} .
$$

The dimensional flux on the diagonal presents the sum of its individual members. So, for the first diagonal (denoted by $<0>$ ) the flux is equal to

$$
\Pi^{<0>}(k, n, r)=H S(0,0, r)=0,
$$

for the second, we have

$$
\Pi^{<1>}(k, n, r)=H S(0,1, r)+H S(1,0, r)=0
$$

for the third

$$
\Pi^{<2>}(k, n, r)=H S(0,2, r)+H S(1,1, r)+H S(2,0, r)=0,
$$

and for the fourth

$$
\Pi^{<3>}(k, n, r)=H S(0,3, r)+H S(1,2, r)+H S(2,1, r)+H S(3,0, r)=3+10 \pi .
$$


The flux in the $n$th diagonal would be calculated in the form of a sum

$$
\Pi^{<n>}(k, n, r)=\sum_{u=0}^{n} H S(u, n-q, r)=\frac{2 r^{n-3}}{\Gamma(n-2)} \sum_{u=0}^{n} \frac{\sqrt{\pi^{u}} \Gamma(u)}{\Gamma(u / 2)} \quad(n \neq 2) .
$$

The flux for the value $n=2$ is calculated on the basis of the function limit value. Respecting that

$$
\frac{\Gamma(u)}{\Gamma(u / 2)}=\frac{2^{u-1}}{\sqrt{\pi}} \Gamma\left(\frac{u+1}{2}\right),
$$

the expression for the flux of the $n$th diagonal, after reordering, can get a new form, and it is equivalent to the expression (2.7)

$$
\Pi^{<n>}(k, n, r)=\frac{r^{n-3}}{\Gamma(n-2)} \sum_{u=0}^{n} 2^{u} \sqrt{\pi^{u-1}} \Gamma\left(\frac{u+1}{2}\right) .
$$

So, for the fifth diagonal $(n=4)$ we get

$$
\Pi^{<4>}(k, n, r)=\sum_{u=0}^{n} H S(u, n-u, r)=r\left(10 \pi+12 \pi^{2}+3\right) .
$$

For the sixth diagonal $(n=5)$ it follows that

$$
\Pi^{<5>}(k, n, r)=\sum_{u=0}^{n=5} H S(u, n-u, r)=\frac{r^{2}}{2}\left(10 \pi+76 \pi^{2}+3\right) .
$$

As the number of diagonals is infinite, the total flux is formed as the series of all diagonal fluxes

$$
\Pi_{H S}(k, n, r)=\sum_{n=0}^{\infty} \Pi^{<n>}(k, n, r)
$$

namely

$$
\Pi_{H S}(k, n, r)=\sum_{n=0}^{\infty} \sum_{u=0}^{n} H S(u, n-u, r)=\sum_{n=0}^{\infty}\left(\frac{2 r^{n-3}}{\Gamma(n-2)} \sum_{u=0}^{n} \frac{\sqrt{\pi^{u}} \Gamma(u)}{\Gamma(u / 2)}\right) .
$$

For example, approximately, the flux for $r=1$ and $r=12$ we obtain

$$
\Pi_{H S}(k, 12,1)=\frac{98641}{1096}+\frac{493205}{18144} \pi+\frac{604099}{9072} \pi^{2}+\frac{99541}{1512} \pi^{3}+\frac{46061}{1260} \pi^{4}+\frac{5143}{378} \pi^{5}+\frac{11}{6} \pi^{6} .
$$

In the expanded form, the total flux has the polynomial structures of members

$$
\begin{aligned}
& \Pi_{H S}(k, n, r)=3+10 \pi+r\left(10 \pi+12 \pi^{2}+3\right)+r^{2}\left(5 \pi+38 \pi^{2}+\frac{3}{2}\right)+ \\
& r^{3}\left(\frac{5 \pi}{3}+\frac{38}{3} \pi^{2}+20 \pi^{3}+\frac{1}{2}\right)+r^{4}\left(\frac{5 \pi}{12}+\frac{19}{6} \pi^{2}+37 \pi^{3}+\frac{1}{8}\right)+ \\
& r^{5}\left(\frac{\pi}{12}+\frac{19}{30} \pi^{2}+\frac{37}{5} \pi^{3}+14 \pi^{4}+\frac{1}{40}\right)+r^{6}\left(\frac{\pi}{72}+\frac{19}{180} \pi^{2}+\frac{37}{30} \pi^{3}+\frac{97}{5} \pi^{4}+\frac{1}{240}\right)+\cdots
\end{aligned}
$$

The diagonal flux of the hyperspherical function can be expressed by the series of the general form 


$$
\Pi_{H S}(v, r)=\sum_{v=0}^{\infty} a_{v} r^{v} .
$$

Here, $v$ is a summing index by which the sequence of the matrix elements from left to right and from above to down along the diagonal is taken into consideration. The polynomial coefficients contain rational numbers and the graded constant $\pi$. The first three coefficients are zero, so they are not included in the summation sequence. Its other values $(v=0,1, \ldots, 13)$ are given in Table 3

The approximation of the series with 16 coefficients in decimal notation has the form:

$$
\begin{aligned}
& \sum_{v=0}^{15} a_{v} r^{v} \approx 0+0+0+34.42+152.85 r+392.25 r^{2}+750.88 r^{3}+1179.92 r^{4}+1599.71 r^{5} \\
& +1929.07 r^{6}+2111.7 r^{7}+2129.23 r^{8}+1999.13 r^{9}+1762.55 r^{10}+1469.03 r^{11}+1163.76 r^{12} .
\end{aligned}
$$

Approximately, the double series leads to the solution that is very close to the exact one. Namely, for the unique radius and reducing to $\infty \sim n=30$ the double series of the diagonal flux gets the following structure:

$$
\begin{aligned}
& \Pi_{H S}(u, n, 1) \approx \sum_{n=0}^{n=30} \sum_{u=0}^{n} H S(u, n-u, r) \\
& =\frac{739975398988375932899873137}{90740578753486268006400000}+\frac{739975398988375932899873137}{27222173626045880401920000} \pi+\frac{238514305877004451811873137}{3581864950795510579200000} \pi^{2}+ \\
& \frac{746729898421689857416906069}{11342572344185783500800000} \pi^{3}+\frac{69115170440329813663374289}{1890428724030963916800000} \pi^{4}+\frac{135020172303774283083377}{986310638624850739200} \pi^{5}+ \\
& \frac{361197664753904472319223}{94521436201548195840000} \pi^{6}+\frac{228408779952457540837}{270061246290137702400} \pi^{7}+\frac{522026543218088839027}{3375765578626721280000} \pi^{8}+ \\
& \frac{644267815110873757}{26791790306561280000} \pi^{9}+\frac{20316656463704383}{6251417738197632000} \pi^{10}+\frac{66173656245467}{170493211041753600} \pi^{11}+\frac{655224179171}{15786408429792000} \pi^{12}+ \\
& \frac{21951111737}{5464525994928000} \pi^{13}+\frac{2769843971}{7806465707040000} \pi^{14}+\frac{29}{1556755200} \pi^{15} \approx 19427,315 .
\end{aligned}
$$

The diagonal dimensional flux is characteristic with coefficients that contain $\pi^{n}$ constant in the degrees of the series members, in contrast to vertical fluxes with the domination of function errors, where $\pi$ and $e$ are constants. The horizontal fluxes, as it was presented in (2.6), contain exponential functions. In the meantime, the total flux for the unique radius is convergent and can be calculated with considerably greater value

$$
\Pi_{H S}(k, n, 1) \approx 19427.858848843922,
$$

while, e.g., for $r=2$ the flux value is substantially greater and its value is obtained as $\Pi_{H S} \approx 1375905492.377$.

\section{Conclusion}

On the basis of the assumption of the recurrent relations (1.2) that exist within the hyperspherical function (1.1), we can calculate a discrete dimensional flux of this function in the domain of natural integer degrees of freedom. Quantitative flux value depends on the nominated value of the hypersphere radius. Meanwhile, as the function $H S(k, n, r)$ is the function of three variables, its dependence is certainly also a variable $k$, respectively, $n$. In this article, we calculated several continual fluxes (2.4) and (2.5), for contour hyperspherical function, on the basis of Ramanujan-Hardy's integral. The dimensional flux calculating with diagonal algorithm is much simpler and faster to perform on a computer, because the total flux is now defined as convergent-graded series and it does not contain special functions as components. In any case its value is 
identical with the fluxes that are calculated on the base of series, i.e., the $H S$ matrix columns, so there is a numerically verified statement that

$$
\Phi_{H S}(k, n, r)=\Omega_{H S}(k, n, r)=\left.\Pi_{H S}(k, n, r)\right|_{r=1} \approx 19427.858848843922 .
$$

The flux calculating procedure originates from Freden when he defined it in 1993 as the series of hyperspherical functions that refer to the degree of freedom $k=3$. In that case, we obtain the solution [20].

$$
\sum_{k=0}^{\infty} H S(k, 3, r)=\sum_{k=0}^{\infty} \frac{\sqrt{\pi^{k}} r^{k}}{\Gamma\left(\frac{k}{2}+1\right)}=e^{\pi r^{2}} \operatorname{erfc}(-r \sqrt{\pi}) .
$$

This function belongs to the family of Mittag Feffler's-type functions, which he developed already in the early 20th century [32]. In any case, this solution is initial for solving the other dimensional fluxes, both for hyperspherical and for hypercubic, in other words the hyper-cylindrical function [33]. With continuous flux in the domain $k, n \in \overline{0, \infty}$, the problem is considerably more complex, because for its defining, the double integration (3.1) must be performed. It is supposed that its value is very close to discrete flux that is obtained on the base of twofold series. Total dimensional continual flux $(k, n \in N)$ of the unit hyperspherical function $H S(k, n, 1)$ is equal to the value of twofold integral

$$
\int_{0}^{\infty} \int_{0}^{\infty} \frac{2 \sqrt{\pi^{k}} r^{k+n-3} \Gamma(k)}{\Gamma(k+n-2) \Gamma\left(\frac{k}{2}\right)} d k d n
$$

and its solution can be looked for on numerical bases.

\section{Author details}

${ }^{1}$ Technical Faculty M. Pupin, University of Novi Sad, Zrenjanin, Serbia ${ }^{2}$ Faculty of Electrical Engineering, University of Belgrade, Belgrade, Serbia ${ }^{3}$ Technical High School, Kragujevac, Serbia ${ }^{4}$ Technical Faculty M. Pupin, University of Novi Sad, Zrenjanin, Serbia

\section{Authors' contributions}

$\mathrm{DL}$, the worked to defined the formula for Hipersferičnu function (HS) and the corresponding orthogonal dijalgonalne and fluxes, as a key research contributes to the work. NC, has verified the analitical formulas and numerical basis. $B D$, using software packages Mathcad and Mathematica radio program on verification of the obtained expressions and corresponding graphical presentations.

$\mathrm{IB}$, is also working on information support of the overall survey about HS function, and thus this part that is hardest probation, and was presented in this paper. All authors read and approved the final manuscript.

\section{Competing interests}

The authors declare that they have no competing interests.

Received: 17 May 2011 Accepted: 1 March 2012 Published: 1 March 2012

\section{References}

1. Conway, JH: Sphere Packing, Lattices and Groups, 2nd edn. pp. 9.Springer, New York (1993)

2. Dodd, J, Coll, V: Generalizing the equal area zones property of the sphere. J Geom. 90, 47-55 (2008). doi:10.1007/ s00022-008-2015-2

3. Manning, HP: Geometry of Four Dimensions. pp. 364. Phillips Press (2010) ISBN-13: 9781445582917, ISBN-10: 1445582910

4. Letić, D, Cakić, N, Davidović, B: The relational translators of the hyperspherical functional matrix, advances in difference equations. J Hindawi 2010, 11 (2010). Article ID 973432

5. Cakić, N, Letić, D, Davidović, B: The hyperspherical functions of a derivative. Abstr Appl Anal 17 (2010). Article ID 364292

6. http://mathworld.wolfram.com/Hypersphere.html 
7. Letić, D, Cakić, N, Davidovic, B, Berkovic, I: Generalisation of the hyperspherical function (monograph to prepare). Technical Faculty M. pp. 1-131.Pupin, Zrenjanin (2010)

8. Bishop, M, Whitlock, A: The equation of state of hard hyperspheres in four and five dimensions. J Chem Phys. 123, 14507 (2005). doi:10.1063/1.1874793

9. Hinton, HC: The Fourth Dimension. Health Research, Pomeroy, WA (1993)

10. Hocking, GJ, Young, SG: Topology. Dover, New York (1988)

11. Maunder, FRC: Algebraic Topology. Dover, New York (1997)

12. Neville, HE: The Fourth Dimension. Cambridge University Press, Cambridge, England (1921)

13. Rohrmann, DR, Santos, A: Structure of hard-hypersphere fluids in odd dimensions. Phys Rev E. 76, 051202http://arxiv. org/abs/0708.2677v2 (2007)

14. Satoshi, M, Watanabe, Y, Ohno, AK: Scaled hypersphere interpolation technique for efficient construction of multidimensional potential energy surfaces. Chem Phys Lett. 414(4-6):265-270 (2005). doi:10.1016/j.cplett.2005.08.063

15. Sloane, AJN: Sequences. A072478, A072479, A072345, A072346, A087299, A087300 and A074457 in "The On-Line Encyclopedia of Integer Sequences".

16. Von, B, Rucker, R: The Fourth Dimension: A Guided Tour of the Higher Universes. Houghtson Miffin, Boston (1984)

17. Sommerville, YMD: An Introduction to the Geometry of $n$-Dimensions. pp. 136. Dover, New York (1958)

18. Wells, D: The Penguin Dictionary of Curious and Interesting Numbers. Penguin Books, Middlesex, England (1986)

19. Joshi, CMJ, Sadan, S: Random walk over a hypersphere. Int J Math Math Sci. 8(4):685-688 (1985)

20. Kabatiansky, GA, Levenshtein, VI: Bounds for packings on a sphere and in space. 1-14 (1978)

21. Letić, D, Davidović, B, Berković, I, Petrov, T: Mathcad 13 in Mathematics and Visualization. Computer Library, Belgrade 1-1196 (2007). ISBN 978-86-7310-398-3

22. Loskot, P, Norman, BC: On monotonicity of the hypersphere volume and area. J Geom. 87(1-2):96-98 (2008)

23. Sasaki, T: Hyperbolic affine hyperspheres. Nagoya Math J. 77, 107-123 (1980)

24. Tu, S-J, Fischbach, E: A new geometric probability technique for an $n$-dimensional sphere and its applications to physics. Math Phys (2001). arXiv: math-ph/0004021v3

25. Woonchul, H, Zhou, A: Short note on the volume of hypersphere. 13 (2006) arXiv: cs/0604056v1 [cs.it]

26. Letić, D, Cakić, N: Srinivasa Ramanujan - The prince of numbers. Computer Library, Belgrade (2010) ISBN 976-86-7310$452-2$

27. Freden, E: Summing a series of volumes (Problem 10207). Am Math Monthly. 100, 882 (1993). doi:10.2307/2324678

28. http://functions.wolfram.com/GammaBetaErf/Erf/21/01/02/03/01/

29. Mitrinović, DS: An Introduction into Special Functions, Scientific Book. pp. 36-37. Belgrade (1991) ISBN 86-23-202247-3

30. Letić, D, Davidović, B, Berković, , Radulović, B: Three Archimedes' bodies. In: Letic D (ed.) Technical Faculty M. pp. 11720. Pupin, Zrenjanin, Belgrade (2010). 1-136

31. Letić, D, Cakić, N, Davidović, B: Mathematical Constants-Exposition in Mathcad. Beograd (2010) ISBN 978-86-87299-04-7

32. http://mathworld.wolfram.com/Mittag-LefflerFunction.html

33. Letić, D, Davidović, B: The dimensional fluxes of the hypercilindrical function. Abstr Appl Anal 2011 (2011). doi:10.1155/ $2011 / 245326$

doi:10.1186/1687-1847-2012-22

Cite this article as: Letic et al:: Orthogonal and diagonal dimension fluxes of hyperspherical function. Advances in Difference Equations 2012 2012:22.

\section{Submit your manuscript to a SpringerOpen ${ }^{\circ}$ journal and benefit from:}

- Convenient online submission

Rigorous peer review

- Immediate publication on acceptance

- Open access: articles freely available online

- High visibility within the field

- Retaining the copyright to your article

Submit your next manuscript at $\gg$ springeropen.com 